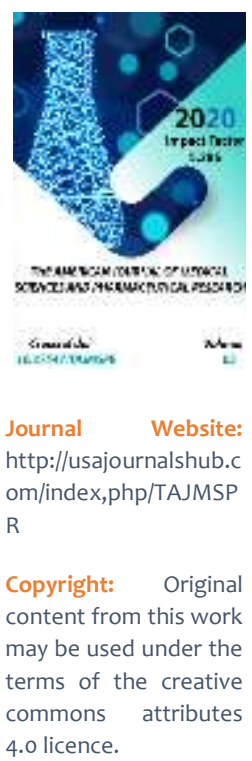

\title{
The Effectiveness Of The Use Of Transtrachial Sanitation In The Treatment Of Patients With Lung Abscess Complicated By Bronchial Fistula
}

\author{
Safoev B.B. \\ Bukhara State Medical Institute, Bukhara, Uzbekistan \\ Khasanov A.K. \\ Bukhara State Medical Institute, Bukhara, Uzbekistan \\ Rakhimov A.Ya. \\ Bukhara State Medical Institute, Bukhara, Uzbekistan \\ Mirsoliev Sh.G. \\ Bukhara State Medical Institute, Bukhara, Uzbekistan
}

\begin{abstract}
The results of the research of 44 patients with lung abscesses complicated by bronchial fistula of various etiology, who were treated in the purulent surgical department in the clinical base of the Bukhara State Institute in 2010-2019, were analyzed. All patients, depending on the method of treatment, were divided into 2 groups: I - comparison group and II - main. The controlled group I consisted of 31 (70.4\%) patients who received traditional (conservative) methods of treatment. The II - main group included $13(29.5 \%)$ patients who, in addition to conservative treatment, underwent transtrachial drainage of a purulent focus with sanitation with antibacterial and diluting drugs (trypsin, chymotrypsin). The analysis of the obtained results revealed that although transtrachial drainage of a purulent focus of the lung is a more effective method than conservative treatment, it also has its drawbacks in the form of complications such as: tracheobronchitis $23.1 \%$, suppuration of soft tissues in the area of microtrachestomy $15.4 \%$, bronchospasm $7.6 \%$, self-extradition of the catheter $23.1 \%$, from the purulent cavity and loss of microdrainage $7.6 \%$.
\end{abstract}

\section{KEYWORDS}

Purulent-destructive lung, fusospirillary, aerugenosa, transtrachial drainage, microtrocar. 


\section{INTRODUCTION}

Literary researches of recent years have noted an increase in the number of patients with severe and complicated forms of acute purulent-destructive lung diseases [Okhunov A.O. 2018, Karimov M.M. 2012, Grudny J, Wiatr E, Gawryluk D, 2013]. The data of the authors' studies show that the percentage of development of pulmonary-pleural complications with lung abscesses ranges from 30 to $70 \%$, and the incidence of gangrenous forms from 28 to $74 \%$ [Bisenkov LN, Okhunov A.O.]. The level of mortality also remains high, in general, with various forms of purulentnecrotic process, from $12.7 \%$ to $77.8 \%$ [Geller D.B., Gostishchev V.K. and etc.]

Acute abscesses and gangrene of the lungs are most often caused by staphylococcus, gramnegative microbial flora and non-clostridial forms of anaerobic infection; fusospirillary flora, previously considered leading in the etiology of gangrenous processes in the lungs, plays a secondary role. Of the strains of staphylococcus with acute suppuration of the lungs, hemolytic and Staphylococcus aureus are most often found, from gram-negative flora - Klebsiella, Escherichia coli, Proteus, Pseudomonas aerugenosa, from anaerobic microorganisms - Bacteroids melaningenicus, B. fragilis, Fusobacterium nucleatum.

The article by Mondoni M. (2019) presents a work describing a new technological approach for the diagnosis of lung lesions - robotic bronchoscopy. Robotic technology, which was introduced and expanded over 20 years ago, has become a major breakthrough in many surgical and endoscopic procedures. The role of classical X-ray research methods and computed tomography in the diagnosis of purulent diseases of the lungs and pleura are considered by the authors (Petukhov V.I., Rusetskaya M.O., Shavrova S.V. 2010).

Siraj O Wali (2012) authors from Saudi Arabia note that most lung abscesses (80-90\%) are now successfully treated with antibiotics, but this conservative approach can sometimes fail. When treatment fails, pulmonary resection is usually recommended. Alternatively, it is recommended that percutaneous transthoracic tube drainage or endoscopically placed drainage may be considered, although both methods remain controversial. Another drainage procedure is endoscopic placement of drains in the cavity of a parenchymal abscess, which was first reported by Metras and Chapin in 1954. Yasnogorodsky O.O., Gostishchev V.K. Shulutko (T.P. 2020) in their article share their experience in providing care to patients with abscesses and lung gangrene. The authors state that the widespread use of minimally invasive technologies in the treatment of lung abscess and postoperative complications has led to a significant decrease in overall mortality by 2,3 times over 42 years.

Recent developments of minimally invasive technologies in the diagnosis and treatment of purulent-inflammatory lung diseases have made it possible over the past decades to slightly reduce the incidence rate, which is confirmed by existing literary publications, but the search for the most effective and minimally traumatic treatment options is extremely relevant.

Purpose of the study: to identify the effectiveness and disadvantages of transtracheal drainage and sanitation in the treatment of patients with lung abscess complicated by bronchial fistula. 


\section{MATERIALS AND METHODS}

The data of examination and treatment of 44 patients with lung abscesses complicated by bronchial fistula of various etiology, who were treated in the purulent surgical department in the clinical base of the Bukhara State Institute in 2010-2019, were analyzed.

All patients, depending on the method of treatment, were divided into 2 groups: I comparison group and II - main. The controlled group I consisted of 31 (70.5\%) patients who received the traditional method of treatment conservative, antibacterial, restorative and symptomatic treatment. In II - the main group included 13 (29.5\%) patients, for whom conservative treatment was supplemented by transtrachial drainage of a purulent focus with the introduction of antibacterial and thinning drugs into the cavity.

The effectiveness of the used and proposed clinical methods for the treatment of suppurative lung diseases was assessed by the duration of bronchopulmonary symptoms, general symptoms of intoxication, the dynamics of the size of the purulentdestructive cavity, and the duration of the total bed-day.

The technique of applying microtrachestomy.

After appropriate premidication in the supine position of the patient, local anesthesia was performed on the anterior surface of the soft tissues of the trachea and along the midline of the neck. After three times skin treatment with $96 \%$ alcohol, a $2 \mathrm{~cm}$ longitudinal incision of the skin and soft tissues was made. A puncture is performed between 2-3 cartilaginous rings of the trachea, with a thin trocar, a tracheostomy is performed by using a microtroacor. Microdrainage is carried out through the lumen of the microtrocar with a lumen with a diameter of $2.0 \mathrm{~mm}$. The microtroacor is removed, the microdrainage is fixed to the skin of the neck using polypropylene sutures, and an aseptic bandage is applied.

The technique of drainage of the abscess cavity through tracheostomy

The position of the patient and the technique of anesthesia was carried out as noted above. A flexible bronchoscope, $5.2 \mathrm{~mm}$ in size, is passed through the nose, larynx and vocal cords under visual control. Infected microdrainage, through a microtracheostomy, is captured with the help of a bronchoscope pin and in the future, under strict visual control, the fistula is brought to the gate and drainage is carried out by introducing the end of the microdrainage into the abscess cavity.

\section{RESULTS AND DISCUSSION}

In all patients, laboratory indicators of signs of endogenous intoxication from peripheral blood (hemoglobin concentration, leukocytosis, ESR, LII, LII, MSM), analysis of the qualitative composition of sputum microflora, and the length of stay of the patient in the hospital were studied as criteria for assessing the condition. At the time of admission and in the course of treatment, the condition of the patients was assessed by clinical signs, according to laboratory and instrumental examination methods, as well as using X-ray research methods.

All patients underwent bacteriological examination of sputum.In most cases, 21 (67.7\%) patients were inoculated with pathogenic staphylococcus, of which 14 $(66.7 \%)$ in the form of a monoculture and in 7 (33.3\%) in associations. In 4 (12.9\%) cases, Pneumococcus was sown, in 2 (6.4\%) cases, colibacillus was sown. Proteus-1 (3.2\%) observations was the next most frequently detected. This was followed by streptococci in 3 (9.6\%) cases. Pseudomonas aeruginosa was inoculated in $2(6.4 \%)$ patients. 
Analysis results of indicators of intoxication of patients' body with lung abscesses complicated by bronchial fistula of the I - the comparison group revealed the following changes (table No. 1). As can be seen from the table, on the first day of treatment, the body temperature of patients averaged 39.1 $\square 0.360 \mathrm{C}$. The content of blood leukocytes was on average $9.6 \square 0.5 \times 109$ / I. Volume c). $0.198 \pm 0.016$ units similarly, an increase in LII and ESR was noted. On the third day of treatment, there was a slight decrease in body temperature indicated from $39.1 \square 0.36$ to $38.6 \square 0.22$, the number of blood leukocytes decreased on average to $8.6 \square 0.25 \times 109 / \mathrm{L}$.
The volume of medium molecules averaged $0.182 \pm 0.009$ units. There was a decrease in LII and ESR indiced to $2.2 \square 0.07$ and $42.3 \square 1.43$, respectively. On the third day of treatment, there was a slight decrease in body temperature indicated from $39.1 \square 0.36$ to $38.6 \square 0.22$, the number of blood leukocytes decreased on average to $8.6 \square 0.25 \times 109 / \mathrm{L}$. The volume of medium molecules averaged $0.182 \pm 0.009$ units. There was a decrease in LII and ESR indicators to $2.2 \square 0.07$ and $42.3 \square 1.43$, respectively. By the seventh day of treatment, the patients of the comparison group with purulent lung diseases maintained an insignificant febrile condition $\left(38.1-0.12^{\circ} \mathrm{C}\right)$.

Dynamics of indicators of intoxication in patients with purulent

lung diseases I - comparison group $(n=31)$

\begin{tabular}{|c|c|c|c|c|c|}
\hline \multirow{2}{*}{ Indicators } & \multicolumn{5}{|c|}{ Observation time } \\
\cline { 2 - 6 } & $\begin{array}{c}\text { Admission } \\
\text { day }\end{array}$ & 3 rd day & 7 th day & 14 th day & 20 th day \\
\hline$t^{\circ}$ body & $39,1 \pm 0,36$ & $38,6 \pm 0,22^{*}$ & $38,1 \pm 0,12 *$ & $37,4 \pm 0,15$ & $36,7 \pm 0,30 *$ \\
\hline L blood \\
$\times 109 / 1$
\end{tabular}

Note: * - differences relative to the data of the previous day are significant $(*-P<0.05$, $* *$ - $\mathrm{P}<0.01, * * *$ - $\mathrm{P}<0.001)$
At the same time, according to all indicators of body intoxication: L, MAW, LII and blood ESR, their further decrease was noted, that is, there was a tendency towards 
normalization - $7.870 .32 \times 109 ; 0.166 * 0.006$; $1.9 \square 0.05 ; 36.6 \square 1.73$ respectively. By the fourteenth day of treatment, these numbers, although they tended to further decrease, however, remained above the norm. With further treatment and observation by the twentieth day, all analyzed parameters of intoxication, except for ESR of blood, were within normal limits.

The average duration of conservative treatment of a lung abscess complicated by a bronchial fistula is $20 \pm 1.5$ days.

The level of microbial contamination, as well as the species composition of microflora from the washout fluid of the II group of patients, revealed the following: In most cases, 8 (61.5\%) patients had pathogenic (Staphylococcus aureus) staphylococcus aureus, in 5 (38.5\%) cases was plated with Pneumococcus. Proteus was next in frequency of detection - 3 (22.0\%) observations. This was followed by enterococci, detected in 2 (15.4\%) cases, streptococci (Streptococcus haemoliticus) in 1 (7.7\%) cases. Pseudomonas aeruginosa was inoculated in $1(7.7 \%)$ patient.

Analysis of the results of indicators of intoxication of patients' body with purulent lung diseases of the II-comparison group revealed the following changes (Table 2 ).

Table 2

\section{Dynamics of indicators of intoxication in patients with purulent}

lung diseases II - comparison group ( $n=13)$

\begin{tabular}{|c|c|c|c|c|c|}
\hline \multirow{2}{*}{ Indicators } & \multicolumn{5}{|c|}{ Observation time } \\
\cline { 2 - 6 } & $\begin{array}{c}\text { Admission } \\
\text { day }\end{array}$ & $3^{\text {rd }}$ day & $7^{\text {th }}$ day & $14^{\text {th }}$ day & $20^{\text {th }}$ day \\
\hline t $^{\text {b body }}$ & $39,5 \pm 0,38$ & $36,9 \pm 0,17^{* *}$ & $36,7 \pm 0,12^{*}$ & $36,6 \pm 0,11$ & $36,5 \pm 0,10^{*}$ \\
\hline L blood & $9,9 \pm 0,36$ & $8,0 \pm 0,25^{*}$ & $7,1 \pm 0,29$ & $6,5 \pm 0,23$ & $6,4 \pm 0,32$ \\
\hline$\times 10^{9} / \pi$ & MAW unit & $0,198 \pm 0,011$ & $0,143 \pm 0,009^{* *}$ & $0,138 \pm 0,006$ & $0,122 \pm 0,007^{* *}, 104 \pm 0,004^{* * *}$ \\
\hline LII unit & $2,5 \pm 0,08$ & $1,6 \pm 0,07^{*}$ & $1,2 \pm 0,08$ & $1,1 \pm 0,06$ & $1,0 \pm 0,05^{* * *}$ \\
\hline ESR mm/h & $48,6 \pm 1,54$ & $38,4 \pm 1,34^{*}$ & $26,3 \pm 1,44^{*}$ & $19,6 \pm 1,13^{* * *}$ & $15,1 \pm 0,60^{* * *}$ \\
\hline
\end{tabular}

Note: * - differences relative to the data of the previous day are significant $(*-P<0.05, * *$ - $P$ $<0.01, * * *-\mathrm{P}<0.001)$

As can be seen from the table, on the first day of treatment, the body temperature of patients averaged 39.5 $\square 0.380 \mathrm{C}$. The content of blood leukocytes was on average $9.9 \square 0.36$ $\mathrm{x} 109$ / I. The volume of medium molecules averaged $0.198 \square 0.011$ units. Similarly, an increase in LII and ESR was noted. On the third day of treatment, a slight decrease in body temperature indicators from 39.5 ото.17 to $36.9 \square 0.14$ was noted, the number of blood leukocytes decreased on average to $8.0 \square 0.25$ $\times 109 / \mathrm{l}$. The volume of medium molecules 
averaged $0.143 \pm 0.009$ units. There was a decrease in LII and ESR indicators to $1.6 \square 0.07$ and $38.4 \square 1.34$, respectively. By the seventh day of treatment, patients in the comparison group with purulent lung diseases retained a slight subfebrile condition $\left(36.7 \square 0.12^{\circ} \mathrm{C}\right)$. At the same time, for all indicators of body intoxication: L, MSM, LII and blood ESR, their further decrease was noted, that is, the tendency towards normalization $-7.1 \square 0.29 \times$ $109 ; 0.138 * 0.006 ; 1.2 \square 0.08 ; 26.3 \square 1.44$, respectively. By the fourteenth day of treatment, these numbers, although they tended to further decrease, however, remained above the norm.

With further treatment and observation by the twentieth day, all analyzed indicators of intoxication were within normal limits. When analyzing control X-ray images and MSCT of the chest, it was found that in group II patients on the day of admission, the sizes of the cavities of the purulent focus of the lung were identical to those in group I. Table 3 shows these figures, different groups on the day of admission did not differ significantly.

Table 3

\section{Dynamics of decreasing the size of abscess cavities of groups I and II.}

\begin{tabular}{|c|c|c|c|c|c|}
\hline \multirow{2}{*}{$\begin{array}{c}\text { Patient } \\
\text { groups }\end{array}$} & \multicolumn{5}{|c|}{ Cavity dimension, cm } \\
\cline { 2 - 6 } & Admission day & 3 days & 7 days & 14 days & 20 days \\
\hline I gr. & $6,9 \pm 0,36 * *$ & $6,7 \pm 0,46 * *$ & $5,8 \pm 0,43 * *$ & $4,9 \pm 0,32 * *$ & $3,8 \pm 0,36 * *$ \\
II gr. & $7,1 \pm 0,72 * * *$ & $5,3 \pm 0,22 *$ & $4,7 \pm 0,27 *$ & $3,3 \pm 0,38 * *$ & $2,5 \pm 0,37 * *$ \\
\hline
\end{tabular}

Notes: - Where * the reliability of differences ( $p$ $<0.05)$ in the size of the foci of destruction between the dynamics according to the terms given in the table.

The dimensions of the cavity of the purulent focus in the course of treatment and in dynamics decreased. It should be noted that the rate of reduction of the cavity size in patients of group I was faster than in group II. By the 18-2oth day of treatment, the size of the cavities during treatment with the use of permanent catheterization of purulent

cavities decreased to $2.5 \pm 0.37 \mathrm{~cm}$, that is, by $35.2 \%$ of the initial size, which in patients of group I during these periods were equal to 3, 8 $+0.36 \mathrm{~cm}, 55.1 \%$. As it can be seen from this, the process of decay of purulent cavities in patients of group II proceeded at a faster pace than in the control group. As can be seen from this, the process of decay of purulent cavities in patients of group II proceeded at a faster pace than in the controlled group. 
The average duration of conservative treatment of a lung abscess complicated by a bronchial fistula using transtrachial drainage is $14 \pm 1.8$ days, which is 3-4 days ahead of the conservative method of treatment.

It should be noted that although there was a positive effect compared with conservative treatment of patients, some complications of this method were noted with the use of permanent catheterization of purulent cavities. Complications were observed in the form of: suppuration of soft tissues in the area of microtrachestomy 2 (15.4\%), acute tracheobronchitis 3 (23.1\%), bronchospasm 1 (7.6\%), in $3(23.1 \%)$ cases during treatment there was an independent extradition of the drainage in the bronchus (exit of the end of the catheter into the bronchus), which was reintroduced into the cavity with the help of a bronchoscope. In 1 (7.6\%) patient, there was an independent loss of microdrainage, where it was necessary to re-establish the drainage in an appropriate way.

Thus, our analysis of the results of a study of patients with a lung abscess complicated by a bronchial fistula revealed the following:

- In a lung abscess complicated by a bronchial fistula, Staphylococcus aureus and Pneumococcus dominate from the pathogenic microflora.

- When using permanent catheterization of the purulent cavity and sanitation with the use of antibiotic therapy, it is an effective method than conservative treatment.

- When used in the treatment complex for endotracheobronchial catheterization of purulent lung cavities with sanitation and antibiotic therapy, the normalization time for all indicators of intoxication is accelerated, and in dynamics there is a decrease in the size of purulent cavities for 4-5 days.
- The average duration of conservative treatment of a lung abscess complicated by a bronchial fistula using transtrachial drainage is $14 \pm 1.8$ days, which is $4-5$ days ahead of the conservative method of treatment.

- Although there are advantages of a positive effect in comparison with only a conservative method of treatment, patients with permanent catheterization of purulent cavities have some complications of this method, such as: suppuration of soft tissues in the area of micro trachestomy up to $15.4 \%$, acute tracheobronchitis $23.1 \%$, bronchospasm $7,6 \%$, in $23.1 \%$ of cases there is an independent extradition of the drainage in the bronchus (exit of the end of the catheter in the bronchus). Up to $7.6 \%$ of patients experience spontaneous micro drainage loss. Which indicates the need to improve the methods of drainage of purulent lung cavities, which do not have the above disadvantages.

\section{CONCLUSIONS}

1. With purulent diseases of the lungs, in patients, mainly from the pathogenic microflora, St. aureus and Pneumococcus dominate.

2. In the treatment of patients with lung abscesses complicated by bronchial fistula, when used in a complex of treatment of endotracheobronchial catheterization of purulent lung cavities with sanitation with antibacterial drugs is a more effective method than conservative treatment.

3. Transtraecheobronchial catheterization of the purulent cavity of the lung is not without drawbacks such as: tracheobronchitis $23.1 \%$, suppuration of soft tissues in the area of microtrachestomy $15.4 \%$, bronchospasm $7.6 \%$, self-extradition of drainage $23.1 \%$, from the purulent cavity and loss of microdrainage $7.6 \%$. 
4. It is necessary to improve the methods of drainage of purulent lung cavities, which do not have complications.

\section{REFERENCES}

1. Blashentseva, S.A. Drainage of acute purulent abscesses of the lungs // Breast and cardiovascular surgery. - 2002. - No. 2. P. 60-64.

2. Grigoriev E.G. Chronic suppurative lung diseases (lecture) // Acta Biomedica Scientifica. 2014. No. 4 (98).

3. Drobyazgin E.A., Chikinev Yu.V., Sudovykh I.Ye. Endoscopic valve bronchial blocking in the treatment of bronchopleural fistulas and air leakage. //Surgery. Journal them. N.I. Pirogov. 2020; (5): 49-57.

4. Okhunov A.O., Babadjanov B.D., Pulatov U.I. The reasons for the generalization of infection in patients with purulent inflammatory diseases of soft tissues against the background of diabetes mellitus /l Bulletin of the Tashkent Medical Academy. No. 4, 2016. S. 89-93.

5. Okhunov A.O., Pulatov U.I., Okhunova D.A. An innovative look at the pathogenesis of surgical sepsis. Results of fundamental research. // Publisher: LAP LAMBERT Academic Publishing RU, 2018.145 p.

6. Petukhov VI, Rusetskaya MO, Shavrova SV The role of classical $X$-ray research methods and computed tomography in the diagnosis of purulent diseases of the lungs and pleura // Problems of health and ecology. 2010. No. 1 (23). -FROM.

7. Safoev B.B., Kurbonov O.M. ,Khasanov A.K., Yarikulov Sh.Sh. The role of bronchoscopy in the treatment of complicated endobronchial ligature fistulas after pulmonary echinococcectomy. // New day in medicine. -2019.-№3 (27) .- P.239-241.

8. Khasanov A.K., Yarikulov Sh.Sh., Mukhamadiev I.Sh., Mirsoliev Sh.G. The problem of acute lung abscess: etiopathogenesis, diagnosis and treatment at the present stage // New day in medicine.2019.-№ 4 (28) .- P.324-328.

9. Khasanov A.K., Yarikulov Sh.Sh., Mirsoliev Sh.G. The current state of the problem of etiopathogenesis and treatment of patients with purulent lung diseases // New day in medicine.-2020.-№3 (31) .- P.149-155.

10. Shoikhet Y.N, Syzdykbaev M.K, Kurtukov V.A, Kapitulin S.Y. Selective transtracheal catheterization of the bronchi in the treatment of acute abscesses and gangrene of the lung // Modern Problems of Science and Education. [Electronic journal]. 2014; (3).

11. Yasnogorodsky OO, Gostishchev V.K., Shulutko A.M. Lung abscess and gangrene: evolution of treatment methods // Surgery news. 2020. No. 2. -S.15-155.

12. Schweigert $M$, Kraus $D$, Ficker JH, Stein HJ. Closure of persisting air leaks in patients with severe pleural empyema - use of endoscopic one-way endobronchial valve.//Eur J Cardiothorac Surg. 2011 Mar; 39 (3): 401-3.

13. Feki W, Ketata W, Bahloul N, Ayadi H Lung abscess: Diagnosis and management // Rev Mal Respir. 2019 Jun; 36 (6): 707-719.

14. Jones NC, Kirk AJ, Edwards RD. Bronchopleural fistula treated with a covered wallstent.//Ann Thorac Surg. 2006 Jan; 81 (1): 364-6. 